\title{
Assessment of knee flexor muscles strength in patients with patellar instability and its clinical implications for the non-surgical treatment of patients after first patellar dislocation - pilot study
}

\author{
Krzysztof Małecki ${ }^{*}$, Jarosław Fabiś ${ }^{2}$, Paweł Flont ${ }^{1}$, Anna Fabiś-Strobin ${ }^{1}$ and Kryspin Niedzielski ${ }^{1}$
}

\begin{abstract}
Background: Biomechanical studies indicate that during outward rotation of the tibia and the valgus knee joint position, the patella is shifted in the lateral direction. After first-time patellar dislocation, the dynamic position of the femur in relation to the tibia plays an important role in joint stability, because the medial stabilizer of the patella (mostly the MPFL) is damaged or inefficient. The most important factor in controlling the rotational movement of the tibia in relation to the thigh are the hamstring muscles. The aim of the study therefore is to determine whether patients with patellar instability have a significant weakness in the knee flexor muscles, which can predispose to recurrent dislocations. This is an important consideration when planning the rehabilitation of patients with first-time patellar dislocation.
\end{abstract}

Methods: The study enrolled 33 patients with confirmed recurrent patellar dislocation, including six patients with bilateral involvement. In the study group, the hamstring muscles (both sides) were evaluated at velocities of 60 and $180 \mathrm{deg} / \mathrm{s}$ for the following parameters: peak torque, torque at 30 degrees of knee flexion, angle of peak torque and peak torque hamstring to quadriceps ratio (H/Q ratio).

Results: In the recurrent patellar dislocation group, a statistically significant weakness in knee flexors was observed for both angular velocities compared to age and gender normative data. No such relationship was observed in the control group of heathy subjects. In patients with one-sided dislocation, no differences were found in knee flexors peak torque, torque at 30 degrees of knee flexion, angle of peak torque or $\mathrm{H} / \mathrm{Q}$ ratio between the healthy and affected limbs for either angular velocity.

Conclusions: In patients with recurrent patellar dislocation, knee flexors strength is decreased significantly in both the unaffected and affected limbs. This may indicate a constitutional weakening of these muscles which can predispose to recurrent dislocations.

\footnotetext{
* Correspondence: krzynormal@wp.pl

${ }^{1}$ Clinic of Orthopaedic and Traumatology, Polish Mother's Memorial Hospital

Research Institute, Rzgowska 281/289, 93-338 Lodz, Poland

Full list of author information is available at the end of the article
}

C C The Author(s). 2021 Open Access This article is licensed under a Creative Commons Attribution 4.0 International License, which permits use, sharing, adaptation, distribution and reproduction in any medium or format, as long as you give appropriate credit to the original author(s) and the source, provide a link to the Creative Commons licence, and indicate if changes were made. The images or other third party material in this article are included in the article's Creative Commons licence, unless indicated otherwise in a credit line to the material. If material is not included in the article's Creative Commons licence and your intended use is not permitted by statutory regulation or exceeds the permitted use, you will need to obtain permission directly from the copyright holder. To view a copy of this licence, visit http://creativecommons.org/licenses/by/4.0/. The Creative Commons Public Domain Dedication waiver (http://creativecommons.org/publicdomain/zero/1.0/) applies to the data made available in this article, unless otherwise stated in a credit line to the data. 
Trial registration: The study was retrospectively registered on ClinicalTrials.gov (NCT04838158), date of registration; 22/03/2021.

Keywords: patella dislocation, Hamstring strength, Isokinetic assessment, Children

\section{Introduction}

Although patients with recurrent, i.e. at least second, dislocation of the patella are qualified for surgical treatment, there is a lack of clear agreement on how to treat those with the first-time dislocation [1-4]. The routine management the first dislocation is conservative treatment and rehabilitation; however, there are no unequivocal guidelines and no data indicating that such treatment prevents recurrence [5-9]. Recurrent dislocations occur in 15 to $72 \%$ of cases of first-time dislocation, depending on the risk factors [10]. The most frequently-mentioned risk factors include patella alta, increased TT-TG distance, trochlear dysplasia, patellar tilt, bilateral occurrence of the dislocation, skeletal immaturity, genus valgus, increased femoral anteversion and familial occurrence $[8,9,11]$. To our knowledge, no analysis of the significance of muscle strength as a risk factor for recurrence of the dislocation has been conducted. While the need to restore the strength of the quadriceps muscle is widely accepted, the role of hamstring strength has been underestimated [12-16].

The dynamic position of the femur in relation to the tibia is influenced by the flexors and extensors of the knee joint. Biomechanical studies indicate that, in a closed kinematic chain, the outward rotation of the tibia and the valgus knee joint position shifts the patella in a lateral direction. Patellar dislocation leads to an injury or stretching of the medial patellofemoral ligament (MPFL); this occurs when the patella moves out of the intercondylar groove, typically during the last 2030 degrees of knee joint extension [17]. The dynamic position of the femur in relation to the tibia in patients after first-time patellar dislocation plays a key role in its stability, because the medial stabilizer of the patella (mostly the MPFL) is damaged or inefficient. As the hamstring muscles are mainly responsible for controlling the rotational movement of the tibia in relation to the thigh, the present study will examine their strength in patients with recurrent patellar dislocation.

The aim of the work is to confirm whether patients with patellar instability have a statistically significant weakness in the knee flexor muscles that can increase the probability of recurrent dislocation. Such knowledge could be of value when performing the rehabilitation of patients with first-time patellar dislocation.

\section{Materials and methods}

The study enrolled 33 patients with confirmed recurrent patellar dislocation, including six patients with bilateral involvement. The study group consisted of 20 girls and
13 boys with a mean age of 16.2 years at the time of the study (range: eight to 17 years, SD 2.7). For the purpose of the study, a control group of 18 healthy subjects (12 girls and six boys) mean 15.7 years (range: 13 to 17 years, SD 1.5), was also recruited. A three-month standard rehabilitation programme with thigh muscle strengthening was performed after the last recurrent dislocation, followed by an individual home exercise programme. Isokinetic testing was performed six months after the incident. Surgical patellar stabilisation was performed in all cases after testing.

The following inclusion criteria were applied: recurrent patellar dislocation, correctly-completed testing protocol, age under 18 years at the time of testing. Subjects with osteochondral fracture and those with a history of knee surgery were excluded.

Each patient underwent an isokinetic examination on the Biodex Multi-Joint System - Pro (Biodex Medical Systems, Inc. New York, USA). In the study group, hamstring muscles (both sides) were evaluated for peak torque (PT), torque in 30 degree of the knee flexion ( $\mathrm{T} 30 \mathrm{deg}$.), angle of peak torque (APT) and peak torque hamstring to quadriceps ratio (H/Q ratio) at velocities of 60 and $180 \mathrm{deg} / \mathrm{s}$. The lower speed $(60 \mathrm{deg} / \mathrm{s})$ was used to reflect the maximum isokinetic resistance used in everyday situations, and the knee can be subjected to extreme loads during sports; therefore, this speed was used to highlight any deficits in the maximum load and to indicate a potential need for correction.

Torque values in $180 \mathrm{deg} / \mathrm{s}$ are well-standardized and commonly-used isokinetic criteria for admission to training after knee injuries and surgery. When measuring PT, a standard parameter used for the isokinetic evaluation of the knees, the knee joint function is also evaluated in terms of its stability. APT is known to change in cases of patellar instability, and its normalization is a reliable indicator of full recovery of knee function in patellar instability, especially when the value is compared with the healthy knee. The H/Q ratio plays a key role in allowing a return to full activity, and in planning an effective correction procedure that ensures an appropriate hamstring/quadriceps balance; in addition, in the case of unilateral changes, the healthy knee can be used as a point of reference to allow for precise correction in the case of existing deficits. Another important parameter is torque at 30 degrees of knee flexion (T $30 \mathrm{deg}$.$) , which indicates the torque value at$ the position where the patella is the most likely to be malpositioned during active knee motion. 
Isokinetic measurements were performed by a trained, experienced physiotherapist. The patients were instructed on methodology of testing. After a warm-up, the patients performed 10 full repetitions of measurements, with the best result being recorded. None of the patients reported pain, apprehension or instability during the isokinetic testing; however most demonstrated a typical positive apprehension test during passive lateralisation of the patella. In cases of unilateral dislocation, no patellar laxity was evident, nor were any apprehension tests positive on the healthy side. The peak torque (PT) of both the test and control limbs were then compared with the normative data for age and sex available in the literature $[18,19]$. The obtained PT values for the control group of healthy subjects were also compared with normative data. In addition, the PT, $\mathrm{T} 30$ deg., APT and $\mathrm{H} / \mathrm{Q}$ ratio values for involved and uninvolved knee flexor strength were compared in the study group. Depending on the data distribution, the nonparametric Wilcoxon's test or the parametric t-test for the related data was applied. Statistical significance was assumed for $p<0.05$. The study was approved by Polish Mother Memorial Hospital Review Board and registered on ClinicalTrials.gov (NCT04838158). Informed consent was obtained and the rights of subjects were protected.

\section{Results}

In the recurrent patellar dislocation group, a statistically significant weakness in knee flexors peak torque was observed for both angular velocities compared with age- and sex-related normative data $(p<0.001)$ (Tables 1,2$)$. In the control group of healthy subjects, no such weakness was observed for either angular velocity $(p=0.67, p=$ 0.29) compared to normative data (Tables 1, 2). In 27 patients with one-sided dislocation, hamstring peak torque of healthy limbs was significantly decreased compared to normative data $(p<$ $0.001, p=0.004)$. However, in these 27 patients with one-sided dislocation, no difference was observed between the healthy and affected limbs with regard to $\mathrm{PT}, \mathrm{T} 30 \mathrm{deg}$., $\mathrm{APT}$ or $\mathrm{H} / \mathrm{Q}$ ratio for either angular velocity (Table 3 ).

\section{Discussion}

Our findings indicate that patients with recurrent patellar dislocation demonstrate significantly decreased knee flexors strength in both unaffected and affected limbs in comparison with normative data. Creating a control group of healthy patients not affected with patellar instability confirmed the validity of the normative data: no differences in peak torque were found between the normative data and the control group.

Our findings also demonstrate that patients with unilateral recurrent patellar dislocation are also subject to bilateral flexors weakness. In such cases, bilateral hamstring muscles weakness may indicate a constitutional weakening of these muscles. In these patients, it is possible to improve the muscular strength of the flexors through intensive rehabilitation. Rehabilitation is especially important in patients after the first-time patellar dislocation when the passive medial knee stabilizers have been injured.

In the rehabilitation of patients with patellar instability, attention is paid only to the strength of the quadriceps muscle; however, the vastus medialis obliquus (VMO), a part of the quadriceps, generates a force vector moving the patella medially [12-16]. Despite this, there is no mention in the literature concerning the possibility of targeted strengthening of the medial hamstrings to counteract the rotational-valgus malalignment mechanism of patellar dislocation.

In cases of multidimensional anteroposterior knee instability with anterior cruciate ligament insufficiency, it is very important to strengthen the hamstrings, which act as active knee stabilizers [20-26]. A similar rotational mechanism has been observed in anteroposterior knee instability and patellar dislocation. The literature describes the coincidence of patellar dislocation with the MCL and ACL injury [27-29]. By enhancing the strength of the knee flexors, i.e. active knee stabilizers, it is possible to limit the number of torsional injuries that can lead to patellar dislocation, which is highly probable in the case of previous MPFL damage. This may suggest that rehabilitation is very important in the treatment of first-time patellar dislocation, and surgical treatment may not always be necessary; however, this is only a pilot study and such findings need further qualification.

Table 1 Results of peak torque at $60 \mathrm{deg} / \mathrm{s}$ (z-value; Wilcoxon's test)

\begin{tabular}{llll}
\hline Peak Torque 60 & $\mathbf{X}$ (mean) & z-value & $p$-value \\
\hline Normative data (for age and sex) & $72.2 \mathrm{Nm}$ & $\mathrm{z}=-5.535$ & $\mathrm{p}<0.001$ \\
knees with recurrent dislocation $n=39$ & $39.7 \mathrm{Nm}$ & $\mathbf{z}=-4.681$ & $\mathrm{p}<0.001$ \\
Normative data (for age and sex) & $73.13 \mathrm{Nm}$ & & $\mathbf{z}=0.0672$ \\
Uninvolved knees $n=27$ & $53.01 \mathrm{Nm}$ & $\mathbf{z}=-1.826$ & \\
Normative data (for age and sex) & $75.5 \mathrm{Nm}$ & \\
Control group of healthy subjects $n=18$ & $66.3 \mathrm{Nm}$ & \\
\hline
\end{tabular}


Table 2 Results of peak torque at $180 \mathrm{deg} / \mathrm{s}$ (z-value; Wilcoxon's test)

\begin{tabular}{llll}
\hline Peak Torque $\mathbf{1 8 0}$ & $\mathbf{X}$ (mean) & z-value & p-value \\
\hline Normative data (for age and sex) & $51.1 \mathrm{Nm}$ & $\mathrm{z}=-2.594$ & \\
knees with recurrent dislocation $\mathrm{n}=39$ & $27.9 \mathrm{Nm}$ & & $\mathbf{z}=-2.881$ \\
Normative data (for age and sex) & $51.9 \mathrm{Nm}$ & & $\mathrm{p}=0.004$ \\
Uninvolved limbs $\mathrm{n}=27$ & $30.6 \mathrm{Nm}$ & $\mathrm{z}=-1.048$ & $\mathbf{p}=0.2937$ \\
Normative data (for age and sex) & $45.9 \mathrm{Nm}$ & & \\
Control group of healthy subjects $\mathrm{n}=18$ & $53.9 \mathrm{Nm}$ & \\
\hline
\end{tabular}

Even so, our results suggest that in patients with the first-time dislocation, it may be advisable to attempt to strengthen the knee flexors before making the decision to perform surgery.

To our knowledge, no previous study has precisely assessed knee flexor strength in patients with patellar dislocation without surgery; however, there is a noticeable trend towards extending the indications for surgery in the first dislocation of the patella. An analysis of flexor strength (peak torque) at speeds of 90 and 240 $\mathrm{deg} / \mathrm{s}$ by Askenberger et al. did not reveal any significant weakening in comparison with the healthy limb, which was also confirmed in the present study [30]. It is important to note that the constitutional weakening of flexors in this group of patients may affect also the healthy limb, and a deficiency can also be observed compared to the control group. In the same publication, the authors provide further arguments for expanding research into the conservative treatment of first-time patellar dislocations. The results of surgical treatment
(MPFL suturing) and conservative management (knee brace and rehabilitation program) in patients after firsttime dislocation were compared during a two-year follow-up period: the authors observed recurrence rates of $22 \%$ after the surgery and $43 \%$ after conservative treatment. Furthermore, non-surgical treatment was associated with more favourable scores on subjective functional scales [30]. Similarly, better functional outcomes in patients after non-surgical treatment were presented by Zimmerer et al. [1].

As this was a pilot study and only a limited number of adolescent patients were available, no power analysis was performed. Our findings need to be supported by cohort studies of patients after first-time dislocation, who underwent strengthening of flexor muscles correlated with the rate of recurrences and isokinetic muscle assessment. Our findings suggest that, based on the published normative data for age and sex and the results obtained in the control group, the weakening of flexors demonstrated by patients might be constitutional,

Table 3 Results of peak torque (PT), torque at 30 degrees of knee flexion (T 30 deg.), angle of peak torque (APT) and peak torque hamstring to quadriceps ratio (H/Q ratio) for 60 and $180 \mathrm{deg} / \mathrm{s}$. (z-value; Wilcoxon's test, t-value; parametric t-test)

\begin{tabular}{|c|c|c|c|c|}
\hline & & $X$ (mean) & statistical value & $p$-value \\
\hline \multirow[t]{2}{*}{ PT 60} & involved limbs $n=27$ & $39.3 \mathrm{Nm}$ & $z=-0.7786$ & $p=0.436$ \\
\hline & uninvolved limbs $n=27$ & $45.1 \mathrm{Nm}$ & & \\
\hline \multirow[t]{2}{*}{ PT 180} & involved limbs $n=27$ & $29.0 \mathrm{Nm}$ & $z=-0.084$ & $p=0.9362$ \\
\hline & uninvolved limbs $n=27$ & $30.7 \mathrm{Nm}$ & & \\
\hline \multirow[t]{2}{*}{ H/Q ratio 60} & involved limbs $n=27$ & $56.7 \mathrm{Nm}$ & $z=-1.585$ & $p=0.114$ \\
\hline & uninvolved limbs $n=27$ & $44.9 \mathrm{Nm}$ & & \\
\hline \multirow[t]{2}{*}{ H/Q ratio 180} & involved limbs $n=27$ & $57.7 \mathrm{Nm}$ & $z=-0.574$ & $p=0.567$ \\
\hline & uninvolved limbs $n=27$ & $50.7 \mathrm{Nm}$ & & \\
\hline \multirow[t]{2}{*}{ T 30 deg. 60} & involved limbs $n=27$ & $25.3 \mathrm{Nm}$ & $z=-0.773$ & $p=0.441$ \\
\hline & uninvolved limbs $n=27$ & $28.2 \mathrm{Nm}$ & & \\
\hline \multirow[t]{2}{*}{ T 30 deg. 180} & involved limbs $n=27$ & $23.5 \mathrm{Nm}$ & $z=-1.63$ & $p=0.103$ \\
\hline & uninvolved limbs $n=27$ & $22.5 \mathrm{Nm}$ & & \\
\hline \multirow[t]{2}{*}{ APT 60} & involved limbs $n=27$ & 63 deg. & $t=1.182$ & $p=0.248$ \\
\hline & uninvolved limbs $n=27$ & 66 deg. & & \\
\hline \multirow[t]{2}{*}{ APT 180} & involved limbs $n=27$ & $48 \mathrm{deg}$. & $t=1.11$ & $p=0.277$ \\
\hline & uninvolved limbs $n=27$ & 52 deg. & & \\
\hline
\end{tabular}


affecting also the healthy limbs, and is not an effect of patellar instability.

Our study could provide guidance to target rehabilitation in patients with first-time dislocation, who have no clear indications for surgery, such as concomitant osteochondral fracture. Isokinetic testing, a repetitive and objective examination, should be used to monitor the progress of rehabilitation, indicated by an increase in hamstrings strength. We propose that first time patella dislocation should be still treated conservatively, and emphasize that quadriceps strengthening exercises should be supplemented by intensive and dedicated knee flexors training in these patients in any case.

\section{Abbreviations}

MPFL: medial patellofemoral ligament; H/Q ratio: peak torque hamstring to quadriceps ratio; TT-TG distance: tibial tuberosity-trochlear groove distance; SD: standard deviation; PT: peak torque; T 30 deg: torque in 30 degree of the knee flexion; APT: angle of peak torque; VMO: vastus medialis obliquus; $\mathrm{ACL}$ : anterior cruciate ligament; MCL: medial collateral ligament

\section{Acknowledgements}

Not applicable.

\section{Authors' contributions}

K.M. - manuscript preparation, data collector, research director, J.F. - research protocol design, advisor. P.F. - manuscript editing, data interpretation. A.F-S. data collector. K.R.N. - research protocol design, supervisor. The authors read and approved the final manuscript.

\section{Funding}

Not applicable.

\section{Availability of data and materials}

Along with ClinicalTrials registration conditions we are ready to share the raw data upon reasonable request. Responsible person: Krzysztof Małecki email: krzynormal@wp.pl.

\section{Declarations}

\section{Ethics approval and consent to participate}

The study was approved by Polish Mother Memorial Hospital Review Board and registered on ClinicalTrials.gov, NCT04838158, date of registration; 22/03/ 2021.

We confirm that informed consent was obtained from a parents and/or legal guardians as participants are under 16 years. Informed consent was also obtained from all participants.

We confirm that all methods were carried out in accordance with relevant guidelines and regulations.

\section{Consent for publication}

Not applicable.

\section{Competing interests}

The authors declare that they have no competing interests.

\section{Author details}

'Clinic of Orthopaedic and Traumatology, Polish Mother's Memorial Hospital Research Institute, Rzgowska 281/289, 93-338 Lodz, Poland. '²epartment of Arthroscopy, Minimally Invasive Surgery and Sports Traumatology, Medical University of Lodz, Żeromskiego 113, 90-549 Lodz, Poland.
Received: 24 April 2021 Accepted: 17 August 2021

Published online: 28 August 2021

\section{References}

1. Zimmerer A, Sobau C, Balcarek P. Recent developments in evaluation and treatment of lateral patellar instability. J Exp Orthop. 2018:5(1):3. https://doi. org/10.1186/s40634-017-0119-z.

2. Nwachukwu BU, So C, Schairer WW, Green DW, Dodwell ER. Surgical versus conservative management of acute patellar dislocation in children and adolescents: a systematic review. Knee Surg Sports Traumatol Arthrosc. 2016;24(3):760-7. https://doi.org/10.1007/s00167-015-3948-2.

3. Erickson BJ, Mascarenhas R, Sayegh ET, Saltzman B, Verma NN, Bush-Joseph $C A$, et al. Does operative treatment of first-time patellar dislocations Lead to increased patellofemoral stability? A systematic review of overlapping Metaanalyses. Arthroscopy. 2015;31(6):1207-15. https://doi.org/10.1016/j.arthro.2 014.11.040.

4. Bitar AC, Demange MK, D'Elia CO, Camanho GL. Traumatic patellar dislocation: nonoperative treatment compared with MPFL reconstruction using patellar tendon. Am J Sports Med. 2012;40(1):114-22. https://doi.org/1 $0.1177 / 0363546511423742$

5. Saccomanno MF, Sircana G, Fodale M, Donati F, Milano G. Surgical versus conservative treatment of primary patellar dislocation. A systematic review and meta-analysis. Int Orthop. 2016;40(11):2277-87. https://doi.org/10.1007/ s00264-015-2856-x.

6. Khan M, Miller BS. Cochrane in CORR $\bullet$ : surgical versus non-surgical interventions for treating patellar dislocation (review). Clin Orthop Relat Res. 2016;474(11):2337-43. https://doi.org/10.1007/s11999-016-5014-x.

7. Liebensteiner MC, Dirisamer F, Balcarek P, Schoettle P. Guidelines for treatment of lateral Patella dislocations in skeletally mature patients. Am J Orthop (Belle Mead NJ). 2017;46(2):E86-96.

8. Jaquith BP, Parikh SN. Predictors of recurrent patellar instability in children and adolescents after first-time dislocation. J Pediatr Orthop. 2017;37(7):48490. https://doi.org/10.1097/BPO.0000000000000674.

9. Balcarek P, Oberthür S, Hopfensitz S, Frosch S, Walde TA, Wachowski MM, et al. Which patellae are likely to redislocate? Knee Surg Sports Traumatol Arthrosc. 2014;22(10):2308-14. https://doi.org/10.1007/s00167-013-2650-5.

10. Magnussen RA, Verlage M, Stock E, Zurek L, Flanigan DC, Tompkins M, et al. Primary patellar dislocations without surgical stabilization or recurrence: how well are these patients really doing? Knee Surg Sports Traumatol Arthrosc. 2017;25(8):2352-6. https://doi.org/10.1007/s00167-015-3716-3.

11. Zhang GY, Ding HY, Li EM, Zheng L, Bai ZW, Shi H, et al. Incidence of second-time lateral patellar dislocation is associated with anatomic factors, age and injury patterns of medial patellofemoral ligament in first-time lateral patellar dislocation: a prospective magnetic resonance imaging study with 5-year follow-up. Knee Surg Sports Traumatol Arthrosc. 2019:27(1):197205. https://doi.org/10.1007/s00167-018-5062-8.

12. Krych AJ, O'Malley MP, Johnson NR, et al. Functional testing and return to sport following stabilization surgery for recurrent lateral patellar instability in competitive athletes. Knee Surg Sports Traumatol Arthrosc. 2018;26(3):711-8. https://doi.org/10.1007/s00167-016-4409-2.

13. Rauschning W, Nordesjö LO, Nordgren B. Isokinetic knee extension strength and pain before and after correction of recurrent patellar dislocation. Arch Orthop Trauma Surg. 1983;102(2):102-6. https://doi.org/10.1007/BF02498724.

14. Oliva F, Ronga M, Longo UG, Testa V, Capasso G, Maffulli N. The 3-in-1 procedure for recurrent dislocation of the patella in skeletally immature children and adolescents. Am J Sports Med. 2009;37(9):1814-20. https://doi. org/10.1177/0363546509333480.

15. Ronga M, Oliva F, Longo UG, Testa V, Capasso G, Maffulli N. Isolated medial patellofemoral ligament reconstruction for recurrent patellar dislocation. Am J Sports Med. 2009;37(9):1735-42. https://doi.org/10.1177/03635465093334 82.

16. Małecki K, Niedzielski K, Flont P, Fabis-Strobin A, Fabis J. Bilateral Hidden Isokinetic Quadriceps Performance before and after MPFL Reconstruction in Pediatric Patients [published online ahead of print, 2020 Jan 6]. J Knee Surg. 2020. https://doi.org/10.1055/s-0039-3402031.

17. Dewan V, Webb MSL, Prakash D, Malik A, Gella S, Kipps C. When does the patella dislocate? A systematic review of biomechanical \& kinematic studies. J Orthop. 2019;20:70-7. https://doi.org/10.1016/j.jor.2019.11.018.

18. Wiggin MM, Jackson R. Quadricep and hamstring peak torque norms in children 6 through 13 years. Pediatr Phys Ther. 2005;17(1):65. 
19. Freedson PS, Gilliam TB, Mahoney T, Maliszewski AF, Kastango K. Industrial torque levels by age group and gender. Isokinet Exerc Sci. 1993;3(1):34-42. https://doi.org/10.3233/IES-1993-3105.

20. LaPrade MD, Kennedy MI, Wijdicks CA, LaPrade RF. Anatomy and biomechanics of the medial side of the knee and their surgical implications. Sports Med Arthrosc Rev. 2015;23(2):63-70. https://doi.org/10.1097/JSA. 0000000000000054

21. Flaxman TE, Alkjær T, Simonsen EB, Krogsgaard MR, Benoit DL. Predicting the functional roles of knee joint muscles from internal joint moments. Med Sci Sports Exerc. 2017;49(3):527-37. https://doi.org/10.1249/MSS. 0000000000001125

22. Cleather DJ. An important role of the biarticular hamstrings is to exert internal/external rotation moments on the tibia during vertical jumping. J Theor Biol. 2018;455:101-8. https://doi.org/10.1016/j.jtbi.2018.07.013.

23. Kittl C, Becker DK, Raschke MJ, Müller M, Wierer G, Domnick C, et al. Dynamic restraints of the medial side of the knee: the semimembranosus corner revisited. Am J Sports Med. 2019;47(4):863-9. https://doi.org/10.1177/ 0363546519829384.

24. Toor AS, Limpisvasti O, Ihn HE, McGarry MH, Banffy M, Lee TQ. The significant effect of the medial hamstrings on dynamic knee stability. Knee Surg Sports Traumatol Arthrosc. 2019;27(8):2608-16. https://doi.org/10.1007/ s00167-018-5283-x.

25. Wu R, Delahunt E, Ditroilo M, Lowery MM, DE Vito G. Effect of knee joint angle and contraction intensity on hamstrings Coactivation. Med Sci Sports Exerc. 2017;49(8):1668-76. https://doi.org/10.1249/MSS.0000000000001273.

26. Morgan KD, Donnelly CJ, Reinbolt JA. Empirical based modeling for the assessment of dynamic knee stability: implications for anterior cruciate ligament injury risk. Conf Proc IEEE Eng Med Biol Soc. 2018;2018:1676-9. https://doi.org/10.1109/EMBC.2018.8512631.

27. Gormeli G, Gormeli CA, Karakaplan M, Gurbuz S, Ozdemir Z, Ozer M. Acute patellar dislocation with multiple ligament injuries after knee dislocation and single session reconstruction. J Pak Med Assoc. 2016;66(6):757-60

28. Askenberger M, Ekström W, Finnbogason T, Janarv PM. Occult intra-articular knee injuries in children with Hemarthrosis. Am J Sports Med. 2014;42(7): 1600-6. https://doi.org/10.1177/0363546514529639.

29. Luhmann SJ, Schoenecker PL, Dobbs MB, Gordon JE. Arthroscopic findings at the time of patellar realignment surgery in adolescents. J Pediatr Orthop. 2007;27(5):493-8. https://doi.org/10.1097/BPO.0b013e318093f4d8.

30. Askenberger M, Bengtsson Moström E, Ekström W, Arendt EA, Hellsten A, Mikkelsen C, et al. Operative repair of medial patellofemoral ligament injury versus knee brace in children with an acute first-time traumatic patellar dislocation: a randomized controlled trial. Am J Sports Med. 2018;46(10): 2328-40. https://doi.org/10.1177/0363546518770616.

\section{Publisher's Note}

Springer Nature remains neutral with regard to jurisdictional claims in published maps and institutional affiliations.

\section{Ready to submit your research? Choose BMC and benefit from:}

- fast, convenient online submission

- thorough peer review by experienced researchers in your field

- rapid publication on acceptance

- support for research data, including large and complex data types

- gold Open Access which fosters wider collaboration and increased citations

- maximum visibility for your research: over $100 \mathrm{M}$ website views per year

At BMC, research is always in progress.

Learn more biomedcentral.com/submissions 